\title{
'The HippocraTORs and TEEs Club' - the practicalities of implementing a local mentorship programme in a large teaching hospital
}

\author{
Author: Srikirti Kodali
}

\section{Aims}

We designed and implemented a mentorship programme for core medical trainees (CMTs) in our trust with a specialty focus based on trainee-defined objectives.

\section{Methods}

We ascertained the future specialty choices of the CMTs in our hospital. Based on these choices, we identified potential suitable mentors in individual specialties and linked the groups of trainees and mentors together. Two to three mentors were recruited for each specialty from clinical and academic backgrounds with at least one specialty registrar and one consultant. We identified some key objectives for the programme in a narrative fashion with qualitative data analysed using inductive thematic analysis using online surveys.

\section{Results}

Seventy-five per cent of CMTs signed up for the programme with $80 \%$ of these already decided on their future specialty. A variety of approaches was adopted by the different mentor and mentee groups, some groups focusing on future academic opportunities while others focusing on opportunities to improve trainees' subspecialty applications, eg by facilitating clinic and quality improvement project opportunities. There was relatively little focus on pastoral issues in our data. Ninety per cent of trainees who have signed up for this programme have met their mentors and colleagues within the interested specialty and have started working on the objectives identified. This was in the form of starting work on an audit together, specialty trainee 3 applications and interview support, attending clinics with their mentors etc. It has been rated highly in its practicality and usefulness as a model mentorship programme.

\section{Conclusion}

Core medical training is often generic in its objectives as a medical training programme and provides an opportunity to identify future specialty choices. Although regional and national networks of mentorship exist, they are less practical in terms of developing a local mentor-mentee relationship. A local mentorship programme fosters an informal relationship with greater ease in developing skills necessary towards future career directions.

\section{Conflict of interest statement}

None. 